\title{
Surveillance alone plays a key role in curbing the overuse of antimicrobials: The major role of antibiotic stewardship
}

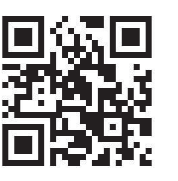

Multidrug-resistant organisms are a growing problem in every facet of medicine globally. With the need to address this ever-increasing threat comes a need for new drugs and new interventions. Infections caused by multidrug-resistant organisms have resulted in high mortality and morbidity all over the world. This is because there are few, if any, pharmaceutical and antimicrobial options to treat them. The pipeline is running dry, and a 'nil-antibiotic' era is approaching. It's a scary thought - no effective antimicrobial agent available anywhere in the world.

We need to conserve what we have, and the answer lies in antimicrobial stewardship. If put into practice, this will assist in curbing the problem of multidrug-resistant organisms. The fight against these pathogens and the struggle to conserve antimicrobials have many facets, but surveillance remains the key.

Antimicrobial stewardship is a team approach and involves many aspects of healthcare, working towards a common goal.

A recent study in the trauma intensive care unit (ICU) at Inkosi Albert Luthuli Central Hospital in Durban, South Africa, highlights the key role that surveillance alone plays in curbing overuse of antimicrobials and reducing multidrug resistance. ${ }^{[1]}$ Surveillance was key to establishing the flora typical of the unit and to choosing empiric therapy, with the help of an antibiogram based on the findings of the surveillance. Patients admitted to the unit were started on empiric therapy when they presented with sepsis. In more than $90 \%$ of cases, empiric therapy proved appropriate. Importantly, there was no need to use initial ultrabroad-spectrum therapy regimens in the form of a carbapenem, a glycopeptide and an antifungal.

Most ICUs around the world employ a de-escalation strategy whereby ultrabroad-spectrum therapy is used and then tailored down or de-escalated once microbiological cultures become available. The Durban study highlights the fact that surveillance and knowledge of local flora plus antimicrobial susceptibility patterns make it possible to use more tailored, narrow-spectrum antimicrobials. Because there is no overuse of broad-spectrum antimicrobials, the emergence of multidrug-resistant pathogens is curbed.

Antimicrobial stewardship is a key component of the multifaceted approach to preventing antimicrobial resistance. Good antimicrobial stewardship involves selecting an appropriate drug and optimising its dose and duration to cure an infection, while minimising toxicity and the conditions that might select for resistant bacterial strains. With such an active surveillance programme and empiric antimicrobial policy, blanket cover is rarely indicated. The empiric choice of antimicrobials should cover the most likely pathogens, endemic to the specific location. It is important to note that this does not refer to all pathogens, and prescriptions cannot be based on uncommon organisms unless the situation dictates the need.

There are many challenges to successful stewardship, but its aims are education, prevention of antimicrobial overuse, and minimising the development of resistance. Pivotal to success are interested clinicians and microbiologists, knowledge of local resistance patterns, and an antimicrobial policy that optimises the choice, dose and duration of therapy.

Almost 70 years ago Sir Alexander Fleming correctly predicted the development of antimicrobials when he stated, 'The intensive research which penicillin has stimulated may bring forth others as good, or even better, or the chemists may be able to modify the penicillin molecule so that its power is increased or its limitations are removed. There is still plenty of scope for further advance.'[2] In the same book (edited by Fleming), however, Porritt and Mitchell made the pessimistic prophecy, 'As with sulphonamides, the prophylactic use of penicillin will carry the risk of breeding resistant strains, a matter of serious practical importance that cannot be dismissed lightly. ${ }^{[3]}$

Unfortunately the indiscriminate use of drugs foreseen by Fleming has promoted the emergence of resistance to even the broadest-spectrum antimicrobials. While his prediction that there is scope for further advance in the discovery of new antimicrobials undoubtedly held true in the first half of the 20th century, the same cannot be said today. There are few new avenues, if any, and strict measures must be implemented to enforce infection control and curb indiscriminate and inappropriate prescribing.

When combined with regular bacterial surveillance, antimicrobial stewardship allows accurate empiric antimicrobial prescriptions with minimal need for de-escalation. This policy may reduce the emergence of multidrug-resistant pathogens, avoiding the need for broad-spectrum antimicrobials and the attendant problems of overuse.

\section{K S S Han}

Y Ramsamy

Department of Medical Microbiology, Nelson R Mandela School of Medicine, University of KwaZulu-Natal, Durban, South Africa; National Health Laboratory Services, KZN Academic Complex, Durban, South Africa

Corresponding author: K S S Han (dr.khine85@gmail.com)

1. Ramsamy Y, Muckart DJJ, Han KSS. Microbiological surveillance and antimicrobial stewardship minimise the need for ultrabroad-spectrum combination therapy for treatment of nosocomial infections in a trauma-intensive care unit: An audit of an evidence-based empiric antimicrobial policy. S Afr Med J 2013;103(6):371-376. [http://dx.doi.org/10.7196/SAMJ.6459]

Fleming A. History and development of penicillin. In: Fleming A, ed. Penicillin. London: Butterworth \& Co., 1946:1-23.

Porritt AE, Mitchell GAG. Prophylactic use of penicillin. In: Fleming A, ed. Penicillin. London: Butterworth \& Co., 1946:105-115.

S Afr Med J 2013;103(6):368. DOI:10.7196/SAMJ.7017 